\title{
Cycling in Newton's Method
}

\author{
Mikheev Serge E. \\ Faculty of Applied Mathematics \& Control Processes,Saint Petersburg State University, 198504, Russia \\ *Corresponding Author: him2@mail.ru
}

Copyright (C2013 Horizon Research Publishing All rights reserved.

\begin{abstract}
Cycling in Newton's method for systems of nonlinear equations in multi-dimensional spaces is researched. The functions of the system have most favorable for convergence properties such as convexity or concavity, no singularity of Jacobi's matrix for the functions and of course existence of the root. It was shown by the counterexample that these properties does not prevent cycling in pure Newton's method while various relaxations of the method have good convergence.
\end{abstract}

Keywords cycling, cycle, convergence, nonlinear equation, iteration

\section{Introduction}

Let us consider a system of linear equations

$$
g(x)=0,
$$

$g: R^{n} \longrightarrow R^{n}$. One of the common methods of obtaining iterative approximations to a solution $\alpha$ of (1) (in other words: $\alpha$ is a root of $g$ ) is the Newton's one (NM)

$$
x^{k+1}=x^{k}-J^{-1}\left(x^{k}\right) g\left(x^{k}\right), \quad k=1,2, \ldots
$$

where $J$ is Jacobi's matrix of function $g$. Equally popular is the simplified Newton's method (SNM), which in contrast to the main method uses the initial matrix $J\left(x^{0}\right)$ instead of $J\left(x^{k}\right)$ for each iteration.

The choice of initial approximation $x^{0}$ lies outside NM and SNM.

In contrast to scalar case the problem of NM convergence in multidimensional one was waiting its hour till 1948. Then Kantorovich has published the theorem about semiglobal convergence of NM and SNM in Banach' spaces. After a short space several close to the theorem results were discovered by Kantorovich himself and his disciple Mysovskikh. The results are presented in [1]. To better understand what problems in NM were left to other researchers we use some other language to describe the results.

There is a key condition in Kantorovich' and Mysovskikh' theorems. If one extracts it then the remaining ones can be regarding as forming a class of functions. Such a class for Kantorovich' theorem [1, p. 680] is a set of functions $g$ defined in some ball centered in initial point $x^{0}, J$ must have derivative $J^{\prime}$ in the ball and in the point $x^{0}$ the continuous linear operator $J^{-1}\left(x^{0}\right)$ exists, such that $\left\|J^{-1}\left(x^{0}\right) J^{\prime}(x)\right\| \leq K$ in the ball. So, the class may be denoted as $\mathcal{K}\left(x^{0}, J\left(x^{0}\right), K\right)$. The key condition of the theorem is $K\left\|J^{-1}\left(x^{0}\right) g\left(x^{0}\right)\right\| \leq 1 / 2$. It provides convergence both NM and SNM for each element of class $\mathcal{K}\left(x^{0}, J\left(x^{0}\right), K\right)$. As the class $\mathcal{K}$ and the key condition contain no estimation of $\left\|\alpha-x^{0}\right\|$ the theorem is not local. From the other hand the key condition contains discrepancy, but $\mathcal{K}$ contains elements with arbitrary large discrepancy, so, the key condition is not valid for whole $\mathcal{K}$. This means that the theorem is not global for $\mathcal{K}$. So, the theorem is semiglobal. Nevertheless practice needs demanded more.

At first, it is hard to obtain and to use a second derivative in multidimensional case. Therefore having lost very little in the power of the theorem, the class $\mathcal{K}$ was enriched up to the class $\mathcal{K}^{+}$by functions with derivatives satisfying Lipschitz' condition with constant $L$. Then the theorem takes the form 'if $g \in \mathcal{K}^{+}\left(x^{0},\left\|J^{-1}\left(x^{0}\right)\right\|, L\right)$ and the key condition $L\left\|J^{-1}\left(x^{0}\right)\right\|^{2}\left\|g\left(x^{0}\right)\right\| \leq 1 / 2$ is valid then for each element of $\mathcal{K}^{+}\left(x^{0},\left\|J\left(x^{0}\right)\right\|, L\right)$ both $\mathrm{NM}$ and SNM generate sequences convergating to $\alpha$ '.

At second, even after a big work to estimate parameters $K$ or $L$ of these classes it is necessary to calculate $g\left(x^{0}\right), J^{-1}\left(x^{0}\right)$ and only to know is the convergence guaranteed or may be here is a chance of divergence. Partly the reason, partly real equations pushed to new classes. For example, Mysovskikh examined the class $\mathcal{M}\left(x^{0}, B, L\right)$ where $B \geq\left\|J^{-1}(x)\right\|$ for all $x$ in the ball. He has proved the convergence of NM for elements of $\mathcal{M}$ satisfying the key condition $L B^{2}\left\|g\left(x^{0}\right)\right\|<2$. So, only the discrepancy should be calculated. Quite another classes where NM and SNM can convergate are proposed in [2]. But the convergence in it is supported also only by local and semiglobal theorems.

Besides practice needs there are additional incentive to find classes with global convergence of the methods. This is the simplicity of global convergence in the scalar case. It was well known yet to Newton that the global convergence in the scalar case both NM and SNM can be provided by

1) condition for method validity

SNM: existence of $g^{\prime}\left(x^{0}\right)$ and $g^{\prime}\left(x^{0}\right) \neq 0$;

NM: $\quad(\forall x) \exists g^{\prime}(x) \neq 0$;

2) conditions for convergence: 
NM \& SNM: root $\alpha$ exists, $g$ is convex or concave;

SNM: $x^{0}$ must satisfy $g\left(x^{0}\right) \geq 0$ under convexity and $g\left(x^{0}\right) \leq 0$ under concavity.

Another incentive is simplicity of global result for modification of the methods by various relaxations, particularly by their limit, when relaxation step tends to zero, version which is finding root $\alpha$ as a solution of the Cauchy' problem $\dot{x}=J^{-1}(x) g(x), x(0)=x^{0}$. Gavurin [3] has found conditions for existence of a limit point $\bar{x}=\lim _{t \rightarrow \infty} x(t)$ of the solution be a root $\alpha$. He has formulated his result by such a way that it has become local. Strengthening one condition and omitting another in Gavurin's theorem we can get the same affirmation for each initial point $x^{0}$ in Banach' space $\mathbf{B}$, i.e. global result. Namely, let 1) $\left.(\forall x \in \mathbf{B})\left\|J^{-1}(x)\right\| \leq \gamma ; 2\right) J^{\prime}(x)$ is bounded in a neighborhood of each $x$ in $\mathbf{B}$. Then $\bar{x}$ exists and equals to $\alpha$.

Despite various relaxations with positive steps were also very fruitful for global results (e.g. [4]) the interest to pure NM and SNM did not die.

The global convergence in $\mathbf{R}^{\mathbf{n}}$ for convex functions $g$ is set [5] when $J^{-1}(x) \geq 0$ for all $x$, where the inequality is understood componentwise. In general case, the elucidation of such a positivity of $J^{-1}$ is very difficult. Checking the conditions of the theorems of semiglobal convergence is somewhat simpler, but the results are only permissions to use NM and SNM from specific initial point, that significantly reduces the theoretical value of the theorems. And a numerical experiment often requires significantly less human efforts than checking the conditions of the convergence theorems. Therefore most of the applications of NM and SNM in the multidimensional case is blindfold 'i.e. without prior analysis of the initial point for the convergence from it'. Therefore, the following question seems to be of the practical interest: does only easy checking convexity of $g$ with the condition of non-singularity of the Jacobi's matrix throughout the multidimensional space guarantee convergence, as in the one-dimensional case?

The analysis of the next example shows that it is, unfortunately, wrong already in $R^{2}$ and even when $J$ satisfies Lipschitz' condition.

\section{Methodology}

Here we name a reflection to finite-dimensional space convex when all its components are convex.

Counterexample (of bad convex reflection).

Let $g: R^{2} \rightarrow R^{2}, g(x)=\left(g_{1}(x), g_{2}(x)\right)$.

We define $g_{1}$ and $g_{2}$ via their graphs, which are constructed as convex slope cylindrical surfaces. Then for each functions the level lines defined by the equations $g_{1}(x)=0, \quad g_{2}(x)=0, \quad$ can be interpreted as guiding lines in the plane of the arguments. We construct each of them as combinations of pairs of convex parabolas and pair of rays with smooth connections in points $y=0, \pm \tau$. Let $x=(y, z), t_{0}>0, L>l>0$ and

$0=h_{1}(x):=\left\{\begin{array}{lr}l y^{2}+t_{0} y-z, & 0 \leq y \leq \tau \\ L y^{2}+t_{0} y-z, & 0>y \geq-\tau \\ \left(2 l \tau+t_{0}\right) y-l \tau^{2}-z, & y>\tau \\ \left(-2 L \tau+t_{0}\right) y-L \tau^{2}-z, & y<-\tau\end{array}\right.$

$0=h_{2}(x):= \begin{cases}l y^{2}-t_{0} y-z, & 0 \leq y \leq-\tau, \\ L y^{2}-t_{0} y-z, & 0<y \leq \tau, \\ \left(-2 l \tau-t_{0}\right) y-l \tau^{2}-z, \quad y<-\tau . & y>\tau,\end{cases}$

It is evident that $g$ has a single root $\alpha=(0,0)$.

Fig. 1 shows the guiding lines under $t_{0}=1, \tau=1$, $L=2.25, l=1.5$. Here the curve (3) is blue and the curve (4) is red.

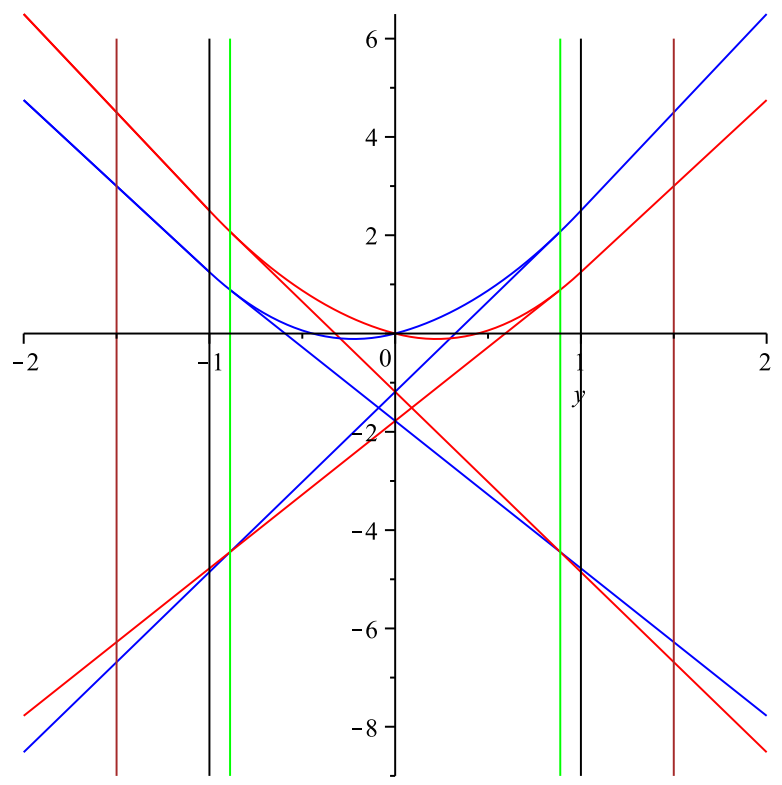

Fig. 1. Unstable cycle.

We rout the forming lines of the cylindrical surfaces so that their projections on the plane of arguments $(y, z)$ are parallel to the axis of symmetry i.e. axis $z$ and the surfaces become convex. The forming lines slopes of both graphs are identical and from interval $\left(0^{\circ}, 90^{\circ}\right)$.

In other words $g_{1}(x)=w h_{1}(x), g_{2}(x)=w h_{2}(x)$ and $w \in(0,+\infty)$. We shall be quite satisfied with $w=1$.

Each step of Newton's method has a simple geometric interpretation in the plane of $x=(y, z)$. Through the current iteration $x^{k}=\left(y^{k}, z^{k}\right)$ a straight line is drawn parallel to the axis of symmetry. At its intersections with lines of levels $g_{1}(x)=0, \quad g_{2}(x)=0$ in points $\left(y^{k}, z_{1}\left(y^{k}\right)\right)$ and $\left(y^{k}, z_{2}\left(y^{k}\right)\right)$, tangents to these lines are drawn. The intersection of the tangents is the next iteration $x^{k+1}$. The second coordinate $z^{k}$ of the current iteration is not involved in the actions.

On Fig. 1 the tangents of both steps have the colors of their curves. Each point on the right green vertical ( $y=$ $\widehat{y} \equiv 8 / 9)$ being chosen as an initial one, i.e. as the first iteration, leads to the single second iteration which is the common point of the left green vertical and two tangents starting from the intersections of the right green vertical with the right branches of curves (3) and (4) in their parabolic parts. The intersection of the tangents to the 
left branches of curves (3) and (4) in the points with abscissa of left green vertical is the third iteration lying on the right green vertical. These two intersections of the tangents are the points of the single unstable cycle on two points. Outside the strip bounded by two black verticals $(y= \pm 1)$ the function $g$ is linear. ( See 3.1.) Extremely left and extremely right brown verticals have abscissas of two point stable cycle $(y= \pm 1.5)$.

\section{Results}

\subsection{Cycles}

Let us define the relationship between the first coordinates $y$ and $v$ of two successive iterations, when $0<y<\tau$. Following geometric interpretation, we consider the equations of the tangents to the lines of levels:

$$
\left\{\begin{array}{l}
z=t_{1}(y)(v-y)+z_{1}(y) \\
z=t_{2}(y)(v-y)+z_{2}(y)
\end{array}\right.
$$

Here $t_{1}, t_{2}$ are tangents of slope angles of tangents to the lines of levels. Differentiating the expressions (3) and (4) yields them:

$$
t_{1}(y)=2 l y+t_{0}, \quad t_{2}(y)=2 L y-t_{0},
$$

where $z_{1}, z_{2}$ are implicit functions defined by equations (3) and (4), respectively:

$$
z_{1}(y)=l y^{2}+t_{0} y, \quad z_{2}(y)=L y^{2}-t_{0} y
$$

Substituting (6), (7) in (5) and excluding $z$ give the equation for the first coordinate $v$ of the next iteration:

$$
\left(2 l y+t_{0}\right)(v-y)+l y^{2}+t_{0} y=\left(2 L y-t_{0}\right)(v-y)+L y^{2}-t_{0} y
$$

or

$$
\left(2 l y+t_{0}\right) v-l y^{2}=\left(2 L y-t_{0}\right) v-L y^{2} .
$$

Hence

$$
\frac{v}{y}=\frac{(L-l) y}{2\left[(L-l) y-t_{0}\right]}=\frac{1}{2-2 \tau_{0} / y} .
$$

Here we introduced the principal parameter

$$
\tau_{0}:=t_{0} /(L-l)
$$

Its geometric sense is the abscissa of the point where two right wings parabolas have parallel tangents.

When the $y$ takes values $\left[0, t_{0} /(L-l)\right)$, the numerator continuously increases monotonically from zero. The negative denominator also grows continuously and monotonically to zero. Consequently, the right side of (8) takes in reverse order all the values of the semiaxis $(-\infty, 0]$. If

$$
y=\widehat{y} \doteq \frac{2 t_{0}}{3(L-l)} \equiv \frac{2}{3} \tau_{0}
$$

the right side is equal to -1 . If $0>y \geq-\tau$ we have

$$
\frac{v}{y}=\frac{(l-L) y}{2\left[(l-L) y-t_{0}\right]}=\frac{1}{2+2 \tau_{0} / y}
$$

which with $y:=-\widehat{y}$ also yields $v / y=-1$. Thus, with choice $y^{0}= \pm \widehat{y}$ and $\tau \geq \widehat{y}$ Newton's method for system $\left(g_{1}(x), g_{2}(x)\right)=(0,0)$ has a cycle at two points with abscissas $\pm \widehat{y}$ and with a single ordinate $\widehat{z}$. Under (5), (6), (7)

$\widehat{z}=t_{1}(\widehat{y})(-2 \widehat{y})+z_{1}(\widehat{y})=-3 l \widehat{y}^{2}-t_{0} \widehat{y}=-\frac{2 t_{0}^{2}}{3} \frac{L+l}{(L-l)^{2}}$.

Obviously, from (8), (10) by the same way we prove convergence of Newton's method when

$$
0<\left|y^{0}\right|<\widehat{y} \wedge\left|y^{0}\right|<\tau
$$

and iterations removal from $\alpha$, when $\tau \geq\left|y^{0}\right|>\widehat{y}$. Consequently, when $\tau>\widehat{y}$ aforementioned cycle can be characterized as unstable and it has practically no chances to be realized on a computer, because the calculation errors will most likely push out from the cycle the iterations to the domain of convergence or divergence. The chances of a cycling under $\tau=\widehat{y}$ and the choice of $\left|y_{0}\right| \geq \widehat{y}$ are also small but much more real.

If $\tau \in\left(\widehat{y}, \tau_{0}\right)$, then at a distance greater than $\tau$ from the axis of symmetry, additionally, there is a stable cycle at two points.

Really. All the cases with positive $y \leq \tau$ are considered yet. Let $y>\tau$. Then to determine $v$ we can continue rays 3 from (3) and 4 from (4) to their intersection (see Fig. 2).

$$
\left(2 l \tau+t_{0}\right) v-l \tau^{2}=\left(2 L \tau-t_{0}\right) v-L \tau^{2} .
$$

This implies

$$
v=\check{y}:=\frac{(L-l) \tau^{2}}{2\left[(L-l) \tau-t_{0}\right]} \equiv\left(2-2 \tau_{0} / \tau\right)^{-1} \tau .
$$

It is clear that inequality $\check{y}<-\tau$ is a sufficient condition to exist cycle on two points with abscissas $\pm \check{y}$. But

$$
\begin{gathered}
\check{y}<-\tau \Longleftrightarrow\left(2-2 \tau_{0} / \tau\right)^{-1}<-1 \Longleftrightarrow \\
\left\{\begin{array}{ccc}
2-2 \tau_{0} / \tau>-1 & \Longleftrightarrow & 3 / 2>\tau_{0} / \tau \\
\tau_{0} / \tau>1 & \Longleftrightarrow & \tau<\tau_{0}
\end{array}\right.
\end{gathered}
$$

In other words $\tau \in\left(\frac{2}{3} \tau_{0}, \tau_{0}\right)$ and see (9). The mutual ordinate of the cycle points is $\check{z}=z_{1}(-\check{y})$.

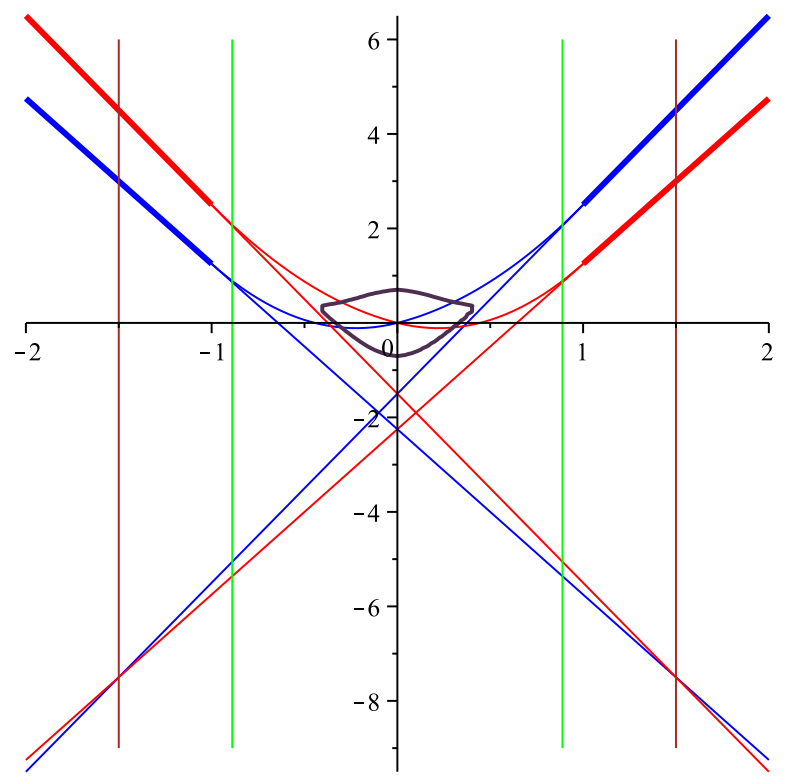

Fig. 2. Stable cycle.

The cycle on $( \pm \check{y}, \check{z})$ is stable. Really. It is evident that if an initial iteration lies in the linearity domain of 
$g$ then the second iteration is one of the cycle points. That implies its stability. Moreover, (8) implies monotone increase of $|v(y)|$ when $y$ runs $(\widehat{y}, 1)$. This means that NM starting with $y \in \widehat{y}, 1)$ will put out iterations moving away from ordinate axis with increasing rate. So, NM' iteration reach the linearity domain for a finite number of steps. Therefore the two point cycle on $( \pm \check{y}, \check{z})$ attracts NM' iteration if and only if abscissa $y$ of initial iteration satisfies $|y|>\check{y}$.

In Fig. 1 the domain of attraction to the stable cycle points is a part of plane outside the strip between two green lines.

\subsection{Newton's method correctness research}

In one-dimensional space for global convergence of Newton's method with a convex function $g$ it is sufficient to require the possibility of constructing a Newtonian iteration in all points in space, i.e. $g^{\prime}(x) \neq 0, \forall x \in R^{1}$. In the counterexample with convex functions the finite decision obviously exists. Hence foresaid part 2) of sufficient conditions for convergency of NM in scalar case continues to be valid. But can NM be non applicable to the function $g$ built in this counterexample in some points of space $R^{2}$ ?

We have found the condition of NM' correctness for counterexample.

Theorem. Let Newton's method be applied to the vector-function $g=h, h$ given by the formulas (3), (4). Then the condition $\tau<\tau_{0}:=\frac{t_{0}}{L-l}$ is necessary and sufficient for the method to be correctly defined in the whole space $R^{2}$.

Proof. Necessity. If $\tau \geq \tau_{0}$, for $x=\left(\tau_{0}, z\right)$ one must use the first case of (3) and the second one of (4). Then

$$
\begin{gathered}
\left.\nabla g_{1}(x)\right|_{x=\left(\tau_{0}, z\right)}=\left(2 l \tau_{0}+t_{0},-1\right) \equiv \\
\equiv\left(2 L \tau_{0}+t_{0},-1\right)=\left.\nabla g_{2}(x)\right|_{x=\left(\tau_{0}, z\right)} \quad \forall z .
\end{gathered}
$$

Consequently, Jacobi's matrix of $g$ is singular in the point $x$ and NM is not applicable.

Sufficiency. We estimate from above the norm of matrix inverse to Jacobi's one. Gradient $\nabla g_{1}(x)$, depending only on the first coordinate $y$, is $\left(z_{1}^{\prime}(y),-1\right)$, function $z_{1}(y)$ implicitly defined in (3) (look (7)). Similarly, $\left.\nabla g_{2}(x)=\left(z_{2}^{\prime}(y)\right),-1\right)$. Consequently,

$$
\begin{gathered}
r \equiv\left\|J^{-1}\right\|=\left\|\left(\begin{array}{cc}
z_{1}^{\prime} & -1 \\
z_{2}^{\prime} & -1
\end{array}\right)^{-1}\right\|= \\
=\mathrm{EIG}^{1 / 2}\left[\left(\begin{array}{rr}
-1 & -z_{2}^{\prime} \\
1 & z_{1}^{\prime}
\end{array}\right)\left(\begin{array}{rr}
-1 & 1 \\
-z_{2}^{\prime} & z_{1}^{\prime}
\end{array}\right)\right]\left(z_{2}^{\prime}-z_{1}^{\prime}\right)^{-1}= \\
=\mathrm{EIG}^{1 / 2}\left(\begin{array}{cc}
\left(z_{2}^{\prime}\right)^{2}+1 & -1-z_{2}^{\prime} z_{1}^{\prime} \\
-z_{1}^{\prime} z_{2}^{\prime}-1 & \left(z_{1}^{\prime}\right)^{2}+1
\end{array}\right)\left|z_{2}^{\prime}-z_{1}^{\prime}\right|^{-1} \leq \\
\leq \frac{\sqrt{2+\left(z_{1}^{\prime}\right)^{2}+\left(z_{2}^{\prime}\right)^{2}}}{\left|z_{2}^{\prime}-z_{1}^{\prime}\right|}
\end{gathered}
$$

Here EIG is the function which gets out the largest eigenvalue of its matrix argument.

Obviously, the modulus of the tangents difference, which is the denominator, is constant in all points $y$ of the set $\{(-\infty,-\tau],[\tau,+\infty)\}$ and is equal to $\left|2 t_{0}-2 \tau(L-l)\right|$. The numerator reaches the global maximum either at $y=0$, or at $y=\tau$.

Indeed, on the set $[0, \tau]$ the expression under the radical is a polynomial of $y$ :

$$
\begin{aligned}
& P(y):=2+\left(t_{0}+2 l y\right)^{2}+\left(t_{0}-2 L y\right)^{2} \equiv \\
& \equiv 2+2 t_{0}^{2}-4(L-l) t_{0} y+4\left(L^{2}+l^{2}\right) y^{2} .
\end{aligned}
$$

It has a positive coefficient at the highest degree, so $P$ reaches a maximum value $\mu>0$ either when $y=\tau$, or when $y=0$. Note that $P(y)=P(\tau) \forall y \geq \tau$. Consequently, we can set $r(x) \leq \sqrt{\mu} /\left[2 t_{0}-2(L-l) \tau\right]$.

Thus, if $\tau \in\left(\frac{2}{3} \tau_{0}, \tau_{0}\right)$ then Newton's method for system $g(x)=(0,0)$ is defined on all $R^{2}$ and has two cycles, one of which is stable. Being defined also on all $R^{2}$ under $\tau=\frac{2 t_{0}}{3(L-l)}$, it has only one unstable cycle. $\diamond$

Note 1. The minimum value of radicand, which is said about in the proof of the theorem, achieved with $y=\frac{L-l}{2\left(L^{2}+l^{2}\right)}=y_{\min }$. Because of the symmetry of the square polynomial relative to its minimizers the condition

$$
\tau<2 y_{\min } \equiv \frac{(L-l) t_{0}}{L^{2}+l^{2}}
$$

ensures that maximum value is achieved on the segment $[0, \tau]$ with $y=0$. Then $\mu=2+2 t_{0}^{2}$.

Note 2. It is easy to show that the Jacobian matrix of the function $g$ introduced in the counterexample has Lipschitz' constant throughout the $R^{2}$. Thus, the function $g$ belongs to the class to which Kantorovich's theorem on the convergence of Newton's method is applicable [1]. Semiglobal restriction of the theorem on the collective parameter $L\left\|J^{-1}\right\|^{2}\left\|g\left(x^{0}\right)\right\| \leq 1 / 2$ for the above found cycle is significantly disrupted, and this is in accordance with world known impossibility to weaken conditions of Kantorovich's theorem. As the object of this theorem is a class of functions, the theorem being applied to the counterexample guarantees convergency of NM in domain $D_{K}$ which is smaller than the mentioned above strip. Containing $D_{K}$ contour $P_{K}(x)=1 / 2$ is drawn in Fig. 2 by black.

Note 3. It is easy to verify that the simplified Newton's method $\left(x^{k+1}=x^{k}-\left(\nabla g\left(x^{0}\right)\right)^{-1} g\left(x^{k}\right)\right)$ has two cycles under the same parameters, each with a pair of points with the same abscissas but with different ordinates.

Note 4. After small modifications this counterexample can be extended to the case of strictly convex functions.

\section{Conclusion}

The counterexample in the article shows that neither convexity nor concavity even together with correctness of pure NM' and SNM' iterations in the whole space and existence of a root guarantee global convergency of pure NM and SNM' in any multidimensional spaces to a root. Thus one easy way to check a priory convergence of NM and SNM is shut. Hardly one can find another set of 
properties easy for verification and close to convergence. As checking positivity of inverse Jacobi's matrix $J^{-1}$ proposed by Ortega is very difficult, it seems best way for application of NM is to enrich it by a kind of relaxation or to use it with care being ready for cycling or divergence.

Collaterally, the counterexample under concrete parameters shows a huge difference between the large real convergence domain and the small theoretical one, which follows from Kantorovich' theorem.

\section{REFERENCES}

[1] Kantorovich L. V., Akilov G.P. Funkcional'nyi analiz (in Russian), Moscow, 1977.
[2] Miheev S. E. Convergence of Newton's method in different classes of functions (in Russian), Computational Technologies, Vol.10, No.3, 72-86, 2005.

[3] Gavurin M.K. Nonlinear functional equations and continuos analoguesof itarative methods (in Russian), Izvestia VUZov, No.5(6), 18-31, 1956.

[4] Mikheev S. E. Method of exact relaxations (in Russian). Computational technologies, Vol.11. No.6, 71-86, 2006.

[5] J. M. Ortega, W. Rheinboldt. Iterative solution of nonlinear equations in several variables. Academic press, NY and London; 1975. 